\title{
Design of 3D printed smart material compatible hand prosthesis
}

\author{
Abdalla M. Omar ${ }^{1, *}$, and Mohamed Hassan ${ }^{2}$ \\ ${ }^{1}$ Department of Mechanical, Aerospace and Civil Engineering, University of Manchester. \\ abdalla.omar@manchester.ac.uk \\ ${ }^{2}$ Department of Mechanical, Aerospace and Civil Engineering, University of Manchester. \\ mohamed.hassan@manchester.ac.uk
}

\begin{abstract}
Every year there are about $3500-5200$ people suffering from upper limb amputations, most of which are wrist disarticulation and transcarpal. This paper investigates current upper limb prostheses and presents the disadvantages of current prostheses, including limited degrees of freedom (DOF), limited range of motion, weight, customizability, and appearance. The proposed design is the first stage of a series of papers that proposes designs that are compatible with shape morphing materials. The use of these materials as actuators allows the development and design of more advanced upper limb prostheses. Therefore, the prosthesis is modelled as needed for patients with transcarpal/wrist disarticulation amputations. The proposed model has 27 degrees of freedom (DOF), reduced weight, low cost, improved appearance, and is printable to fit.
\end{abstract}

\section{Introduction}

Orthopaedic prosthetic devices are external medical devices designed to substitute missing skeletal parts of the body. According to the World Health Organisation (WHO), more than 40 million people need a prosthetic device, but only $5-15 \%$ of them will have access to one [8]. Globally, the orthopaedic prosthetic devices market is expected to grow at a rate of 5\% till 2027 and it is expected to represent 3 billion dollars by 2027. Among the different orthopaedic prosthetic devices, hand prostheses are the most common type of devices for work-related amputations worldwide.

The design and manufacture of upper limb prosthetics depends on the amputation case. Figure 1 highlights the nomenclature used for different upper limb amputation cases. Two classes of amputation are considered. The first corresponds to amputations that take place through the length of the bone such as Transhumeral (b), Transradial (d) and Transcarpal (f). The second class corresponds to amputations at the ends of the bones such as Shoulder disarticulation (a), elbow disarticulation (c) and wrist disarticulation (e) [2]. A study reports that the upper limb amputations: $16 \%$ trans-humeral, $12 \%$ trans-radial, $2 \%$ forequarter, $3 \%$ shoulder disarticulation, $1 \%$ elbow disarticulation, $2 \%$ wrist disarticulation,

\footnotetext{
* Corresponding author: Abdalla.Omar@manchester.ac.uk
} 
$61 \%$ transcarpal, and 3\% bilateral limb loss [2]. Therefore, the case of interest will be transcarpal and wrist disarticulations.

Functionality, cost, and lack of personalisation are the main problems associated to commercially available lower limb prosthesis. Additive manufacturing, also known as 3D Printing, emerged as the ideal technology to produce affordable personalised prostheses. This technology, which allows the fabrication of prosthesis based on the patient's anatomy, has been used to fabricate "passive" devices equipped with DC motors, making the final prosthesis heavy, costly and limited in terms of functionality [6].

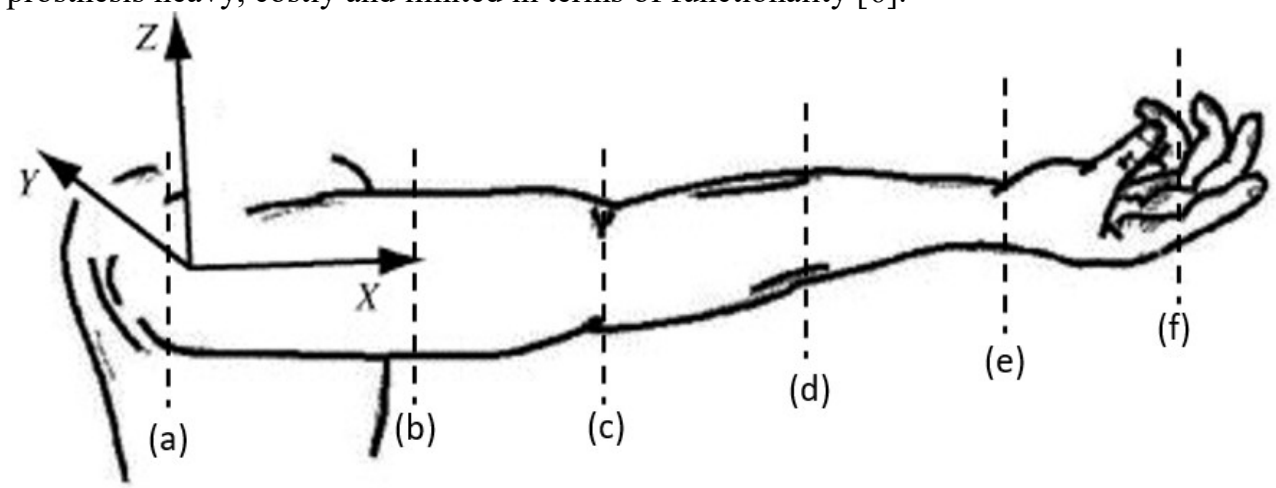

Figure 1. Model of a human hand with different amputation locations[3]

At the University of Manchester, we started a major project aiming to 4D print a full functional upper limb prosthesis. This paper presents preliminary results related to the design, materials, and degrees of freedom of the upper limb prosthesis that will be built on with the use of electro-active shape changing materials.

\section{Methodology}

\subsection{Design}

The wrist has 2 possible movements for the transverse axis, flexion, and extension, and in the longitudinal plane there is ulnar and radial flexion. All these movements depend on the carpal joints along the ulna and radius. The hand has also the same movements, and defined ranges of motion as shown in Figure 3. Based on these movements it is important to define an overall model of the hand's kinematics, critical to design a hand prosthesis. Different models considering different degrees of freedom (DOF) have been proposed $[1,4,7,10]$. Where the more degrees of freedom are considered the lower is the error when using specific grasps (e.g. circular, prismatic). Therefore, a model considering 24 DOF and providing very accurate dexterity and manipulation is commonly used $[4,10]$. Whereas, Morecki proposed a novel kinematic model of the hand based on $22 \mathrm{DOF}$, including the DOFs of the hand and 3 DOF for the wrist $[1,7]$. This is due to an assumption where any motion which is $</ 5$ degrees is a slack and that motion is disregarded. This is the model considered in this research work due to its simplicity and rigour. 


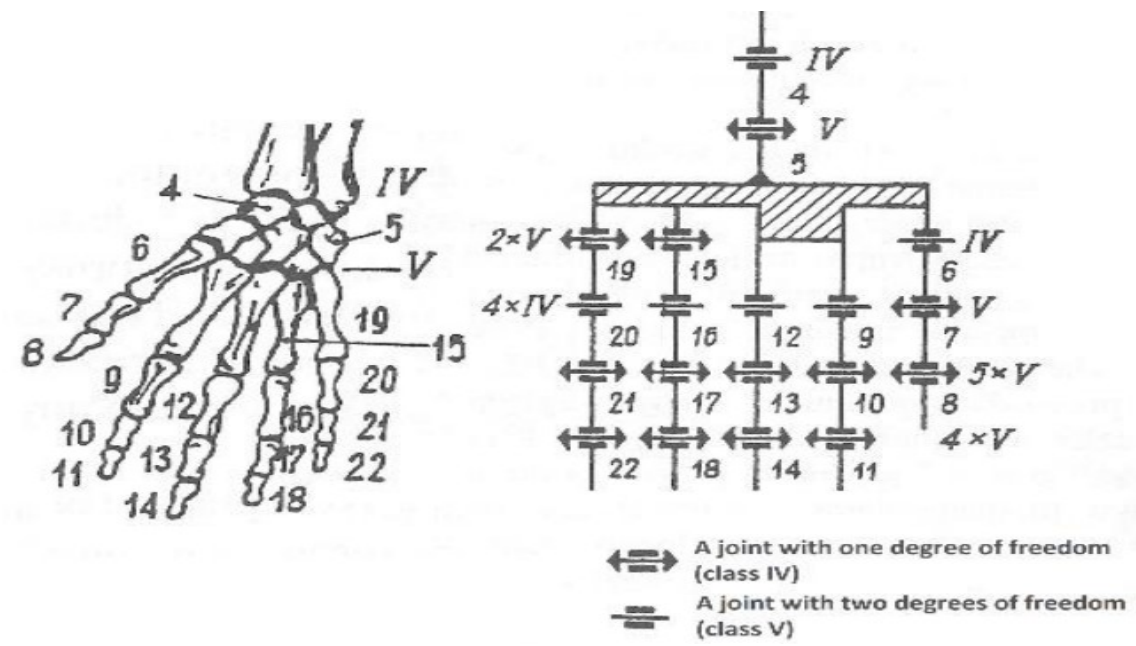

Figure 2. Morecki's kinematic model of the hand $[1,7]$

The hand prosthesis was designed in Dassault systems-Solidworks considering some anatomical measurements were used from literature and then scaled respectively to produce the hand. The hand-wrist connection has a ball-socket joint allowing the wrist to move in all directions. The metacarpophalangeal joints were modified to have 2 DOFs by considering a pivot joint but with a smaller accessory in the inside which allows abduction/adduction movements. The remaining finger proximal and distal joints are basic pivot joints with a small screw acting as a connector. The cad model of the designed hand model is presented in Figure 4.

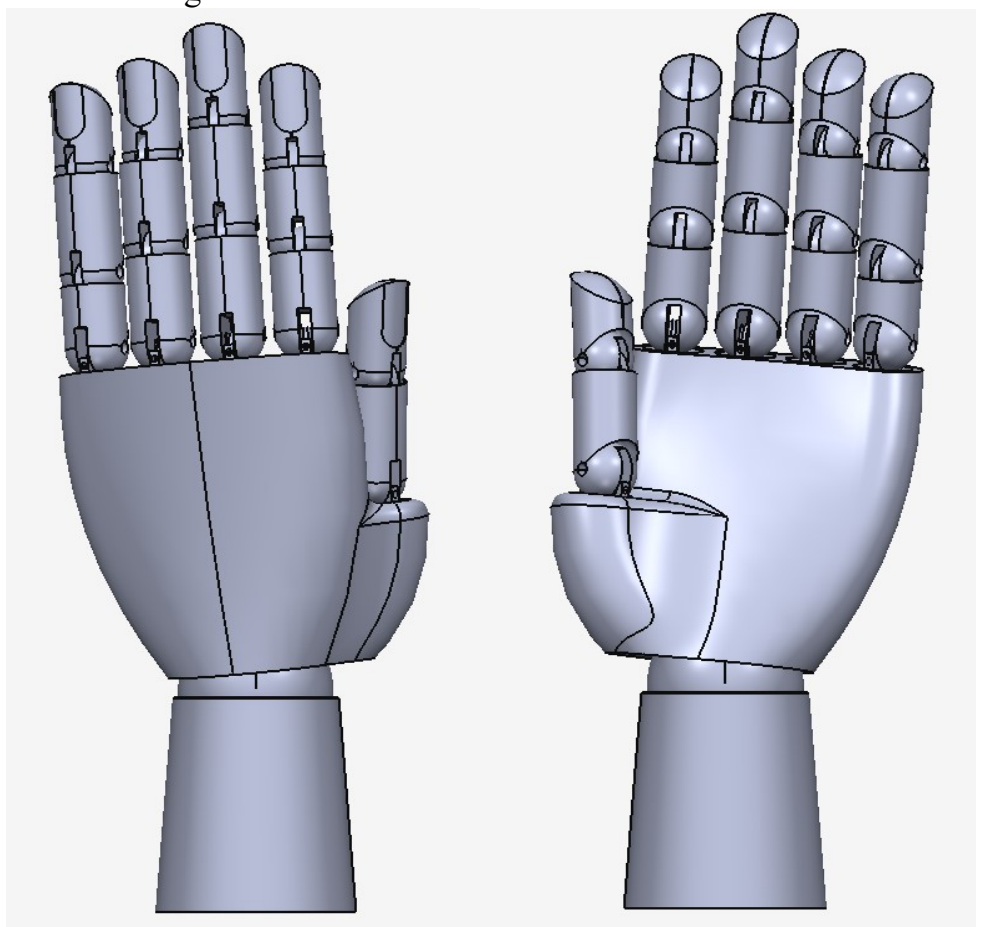

Figure 3. CAD model of the prosthesis; Dorsal view (left), Palmar view (right) 


\subsection{Fabrication}

Moreover, SUNLU-PLA Plus dog bone structures were produced using Extrusion-based 3D printer. The Structures were designed using ASTM D638-14 standard. This standard is specific for testing the tensile properties of plastics. Structure was designed using Type V specimen dimensions, it was printed layer by layer with $100 \%$ infill. The same printing parameters were used to produce the hand; however, the infill percentage was reduced to $15 \%$ for the hand. The printing parameters are presented in Table 1 below:

Table 1. Printing parameters

\begin{tabular}{ll}
\hline Parameters & Values \\
\hline Layer height $(\mathrm{mm})$ & 0.15 \\
First layer height $(\mathrm{mm})$ & 0.27 \\
Extrusion width $(\mathrm{mm})$ & $0.4 \pm 0.05$ \\
Nozzle Temperature $\left({ }^{\circ} \mathrm{C}\right)$ & 205 \\
Bed temperature $\left({ }^{\circ} \mathrm{C}\right)$ & 50 \\
Printing speed $(\mathrm{mm} / \mathrm{s})$ & 60 \\
Travel speed $(\mathrm{mm} / \mathrm{s})$ & 80 \\
Infill $(\%)$ & $100 / 15$ \\
Retraction length $(\mathrm{mm})$ & 1.5 \\
Retraction speed $(\mathrm{mm} / \mathrm{s})$ & 30 \\
\hline
\end{tabular}

\subsection{Mechanical tensile test}

The mechanical properties of the PLA plus was evaluated using the uniaxial tension tests. The tests were carried out using an SAUTER TVL [Manual Crank Handle Test Stand] (Sauter GmbH, UK). The machine is fitted with a $500 \mathrm{~N}$ load cell and two AC 01R Fine Point Clamps that are fitted to the platform and the loadcell. The 5 tensile parts were tested in a dry state to $\sim 2 \%$ strain which is the yield point. The acquired data is presented in the form of a table alongside the respective average values.

\section{Results and discussion}

\subsection{Design and fabrication}

The hand model shown in Figure 4 has one ball and socket joint in the wrist with 3 DOF, five alternative pivot joint for all of the metacarpophalangeal joints each with 2 DOF, and 9 pivot joints for the remaining proximal and distal joints with 1 DOF. This results in a hand model with a total of 22 DOF. The length measurements of the hand are taken from the joints, and radius measurements are of all joints in the respective part. All of which are listed in Table 2. In addition, a sample of the printed index finger is presented in Figure 4.

Table 2. Length and radius measurements of the hand

\begin{tabular}{lll}
\hline Part & Length $(\mathrm{mm})$ & Joint Radius $(\mathrm{mm})$ \\
\hline Thumb & 70 & $9.1 / 9.7 / 9.7$ \\
Index finger & 91.72 & $9.1 / 9.7 / 9.7$ \\
Middle finger & 106.38 & $9.1 / 9.7 / 9.7$ \\
Ring finger & 97.02 & $9.1 / 9.7 / 9.7$
\end{tabular}




\begin{tabular}{lll} 
Little finger & $91 . .61$ & $9.1 / 9.7 / 9.7$ \\
Palm & 99.43 & 33.61 \\
Wrist & 75.45 & 22.2 \\
Overall & 281.26 & 33.61 \\
\hline
\end{tabular}

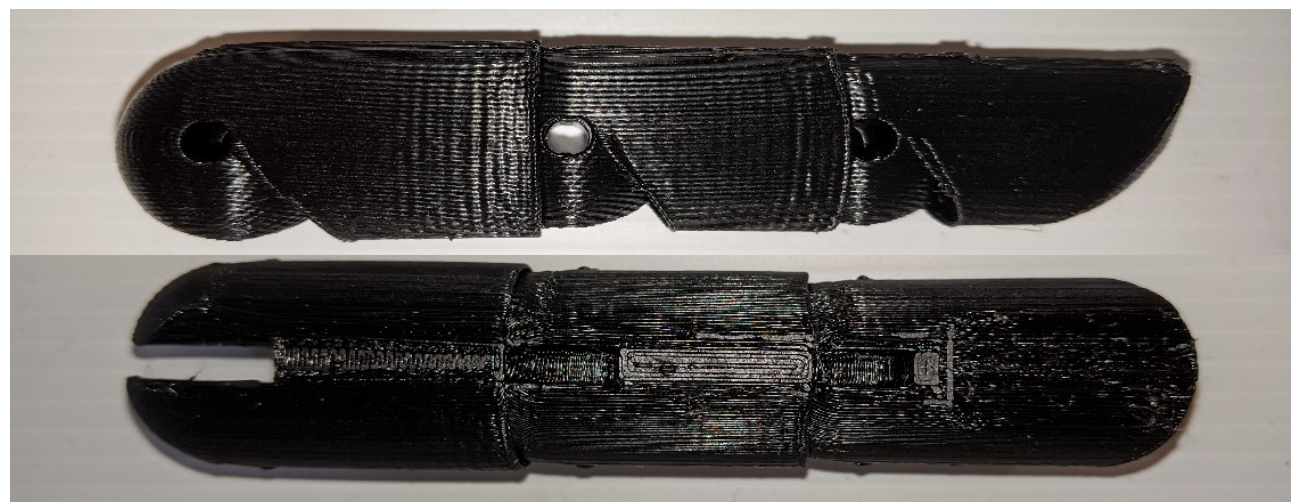

Figure 4. Assembled 3D printed index finger prosthesis.

Considering the use of PLA Plus as the 3D printer filament material, the weight of the full hand prosthesis is $220 \mathrm{~g}$ including all the connections. The filament roll is 335 meters and costs $\sim 21 £$, and the length used to print the whole prosthesis is estimated to be 73.3 meters in length. Therefore, the material cost is approximately $4.60 £$. The operational costs can also be considered such as (e.g. printer cost, printing time, electricity, maintenance and more); However, there are no accurate models to do that, and so an approximation is made based on the following (printed object weight, printing time, electricity tariff, printing power, printer lifetime, time used) and an approximate value of $1.84 £$ is approximated during the printing of this prostheses.

\subsection{Mechanical testing}

Using the SAUTER TVL [Manual Crank Handle Test Stand] to test the dog bone specimens according to the ASTM D638-14 Type V standards were done. Through designing the specimen in the correct dimensions and were measured afterwards to make sure they did not have any defects or deviations from the designed model. Furthermore, the standard specifies the use of 5 different tests to acquire accurate and precise results as seen in Table 3. The testing stand was set to the peak setting to identify the maximum force when reaching the plastic region and was stopped at values of $2 \%$ strain by calculating the displacement required beforehand.

Table 3. Tensile Mechanical properties of PLA Plus

\begin{tabular}{llllll}
\hline Test & Force $(\mathrm{N})$ & Displacement $(\mathrm{mm})$ & Stress $(\mathrm{MPa})$ & Strain $(\%)$ & Modulus $(\mathrm{GPa})$ \\
\hline Test 1 & 502.3 & 1.48 & 52.707 & 0.0233 & 2.261 \\
Test 2 & 498.5 & 1.41 & 52.308 & 0.0222 & 2.355 \\
Test 3 & 491.8 & 1.44 & 51.605 & 0.0226 & 2.275 \\
Test 4 & 504.2 & 1.37 & 52.906 & 0.0215 & 2.452 \\
Test 5 & 489.4 & 1.42 & 51.353 & 0.0223 & 2.296 \\
Average & 497.2 & 1.42 & 52.176 & 0.0224 & 2.328
\end{tabular}


However, there are multiple limitations in this approach as the use of a manual stand means that the strain rate is not fixed as when using an automated mechanical testing machine, which might result in some inaccuracies. Also, the point in which the tests were stopped are based on the hand calculations and approximations made to when necking might start in the small cross-section of the specimen.

The results mainly were used to evaluate the materials mechanical behavior and ensuring that a standard material like PLA can withstand forces up to $500 \mathrm{~N}$, as a maximum force of 538N must be withstood and the material used here starts to yield at that point [8]. Presenting a mostly safe elastic behavior in that range.

\section{Conclusion}

In conclusion, the work presented highlights the challenges and the desired design specifications by upper limb amputees for high quality prostheses. The significance of 4D printing is highlighted and how current DOF limitations can be ignored if such actuators are utilized. Then, the design, fabrication, and testing methodology are presented. The results highlight the 22 DOF achieved in this model using different joints, the cheap cost for a prosthesis skeleton of $6.44 £$, and a weight of $220 \mathrm{~g}$. Further work will build on this design by equipping Shape changing materials.

\section{References}

1. al., A.M.e., Teoria mechanizmów i manipulatorów. (2002)

2. A. Jette, C. Spicer, and J. Flaubert, (2017),

3. E. Pennestrì, R. Stefanelli, P. Valentini, and L. Vita, J. Biomech, 40(6): p. 13501361, (2007).

4. F. Chen Chen, A.F., M. Mousavi, E.P. Ambrosio, S. Appendino, A. Battezzato, D. Manfredi, F. Pescarmona, B. Bona, ICES, (2011)

5. Flashforge, (2020)

6. L. Resnik, M. Meucci, S. Lieberman-Klinger, C. Fantini, D. Kelty, R. Disla, and N. Sasson, Arch Physl Med Rehab, 93(4), pp.710-717, (2012)

7. L. Jaworski, and R. Karpiński, J. Technol. Exploit. Mech. Eng, 3(1), pp.28-33, (2017)

8. M. Hichert, A. Vardy, and D. Plettenburg, Prosthet and Orthot Int, 42(1), pp.8492, (2017)

9. Organization, W.H., $S(2015)$

10. S. Cobos, M. Ferre, Uran Sanchez M.A, J. Ortego, and C. Pena, IROS, (2008) 